\title{
Analysis of Single Synthetic Fibers Using a Portable Total Reflection X-ray Fluorescence Spectrometer
}

Yawara SENGOKU,* Yoshie TOKUOKA,* Hibiki KOMATSU,** Yoshinori NISHIWAKI, ${ }^{* * *}$ and Shinsuke KUNIMURA* ${ }^{\dagger}$

*Department of Industrial Chemistry, Tokyo University of Science, 1-3 Kagurazaka, Shinjuku-ku, Tokyo 162-8601, Japan

**TOSA Innovative Human Development Programs, Kochi University, 2-5-1 Akebono-cho, Kochi 780-8520, Japan ***Division of chemistry, Faculty of Education, Kochi University, 2-5-1 Akebono-cho, Kochi 780-8520, Japan

$\dagger$ To whom correspondence should be addressed.

E-mail: kunimura@rs.tus.ac.jp 


\begin{abstract}
In this study, single synthetic fibers obtained from several textile products were analyzed by a portable total reflection X-ray fluorescence spectrometer. Characteristic elements, which would originate from materials such as catalyst, delustering agent, and dye used for manufacturing synthetic fibers, were detected from the single synthetic fiber samples, and the difference in the types of characteristic elements among the single synthetic fiber samples was observed.
\end{abstract}

Keywords identification, portable, single fiber, synthetic fiber, X-ray fluorescence 


\section{Introduction}

Fragments of textile fibers collected at a crime scene and those collected from a suspect and a crime victim can play a key role in criminal investigation. For example, a case that the criminal and a knife used in the crime were specified by detecting fibers originating from the crime victim's clothing from the knife was introduced in ref. 1. Therefore, in order to determine whether a fiber sample originates from a suspect (or a crime victim), a method to distinguish the difference among fibers is needed.

$\mathrm{X}$-ray fluorescence (XRF) analysis and total reflection X-ray fluorescence (TXRF) analysis make it possible to perform non-destructive elemental analysis of textile fibers. Prange et al. ${ }^{2}$ applied TXRF analysis to single textile fiber analysis. Koons ${ }^{3}$ conducted energy dispersive XRF analysis of carpet fibers. Nishiwaki et al. ${ }^{4}$ performed XRF measurements of single white polyester fibers obtained from trunk mats and clothing using synchrotron radiation, and they used the difference in the types of elements and the ratio of intensities of fluorescent X-ray peaks of elements for distinguishing the difference among polyester fibers. $\mathrm{TiO}_{2}$ is commonly used as delustering agent for polyester fiber, ${ }^{1,5}$ and $\mathrm{Zr}^{6}$ and $\mathrm{Nb}^{1,6}$ originating from impurities in $\mathrm{TiO}_{2}$ were detected from polyester fiber samples by using synchrotron radiation. Takahara et al. $^{7}$ performed TXRF measurements of single black polyester fibers obtained from trunk mats, and they used principal component analysis for the purpose of the classification of these fibers. As described above, the information about elements originating from materials used for manufacturing synthetic fibers can contribute to distinguishing the difference among synthetic fibers with similar colors.

In order to conclude that a textile fiber sample originates from a suspect (or a crime victim), multiple types of information such as chemical component and color obtained from the fiber sample should be compared with those obtained from fiber 
samples from the suspect's textile product (or the crime victim's textile product). Therefore, multiple types of analytical methods should be employed for the identification of fibers. For example, microspectrophotometry is used for comparing colors of fiber samples whose morphologies and color tones observed by an optical microscope are similar to one another, and fourier transform infrared (FT-IR) microscopy is used for investigating materials of fibers. ${ }^{1}$ Raman spectroscopy is used for non-destructive analysis of fibers. ${ }^{8}$ However, it is not desirable to obtain multiple types of information from all textile fiber samples using multiple analytical methods because the time required for identification of fibers becomes long. Therefore, screening for textile fiber samples needed to be analyzed by multiple methods for performing highly accurate identification should be performed for reducing the time required for identification of fibers. Textile fiber samples, whose origins are obviously different from a suspect's textile product (or a crime victim's textile product), would be determined by only comparing the types of detected elements even when colors and morphologies of these fibers are similar to those of fibers originating from the suspect (or the crime victim). Therefore, qualitative XRF (or TXRF) analysis can be used for screening for fiber samples that are needed to be analyzed by other methods for determining whether these fiber samples originate from a suspect's textile product (or a crime victim's textile product). Because multiple elements are detected in one measurement when using energy dispersive XRF (or TXRF) analysis, rapid screening for fiber samples can be performed.

A portable TXRF spectrometer ${ }^{9}$ was developed in 2006. This portable TXRF spectrometer has been improved since 2006, and a detection limit of 8 pg $^{10}$ was achieved for $\mathrm{Cr}$ in the dry residue of a solution sample. Recently, this portable spectrometer was applied to elemental analysis of single hog hairs colored with 
commercially available hair dye products. ${ }^{11}$ This highly sensitive portable spectrometer can be applied to on-site elemental analysis of single textile fiber samples. By performing on-site screening for fiber samples, the time required for identification of fibers using multiple analytical methods will be reduced. In this study, single synthetic fibers obtained from several textile products were analyzed by the portable TXRF spectrometer in order to discuss the possibility that this portable spectrometer can be applied to on-site screening for fiber samples. Single polyester fibers, a single acrylic fiber, and a single nylon fiber were analyzed because these types of synthetic fibers are widely used for clothing.

\section{Experimental}

In this study, strands of white polyester fibers, which were referred to as polyester fiber A, polyester fiber B, polyester fiber C, and polyester fiber D, were obtained from four different textile products. A strand of polyester fiber A was obtained from a semi dull yarn. Strands of polyester fibers B and D were obtained from two different spun threads, and a strand of polyester fiber $\mathrm{C}$ was obtained from a filament thread. Strands of black polyester fibers, which were referred to as polyester fiber $\mathrm{E}$ and polyester fiber F in this study, were obtained from two different spun threads. A single white acrylic fiber was obtained from a yarn, and a single black nylon fiber was obtained from clothing. Sewing threads used for obtaining strands of polyester fibers B, C, and F were manufactured by the same Japanese company, and those used for obtaining strands of polyester fibers D and E were manufactured by the same Japanese company. Single fiber samples described above were analyzed by the portable TXRF spectrometer. The setup of the portable spectrometer used in this study was explained in a previous paper. $^{12}$ A tube voltage and a tube current of an X-ray tube $50 \mathrm{kV}$ Magnum (Moxtek 
Inc., Orem, UT, USA) having a tantalum anode were set to $25 \mathrm{kV}$ and $0.2 \mathrm{~mA}$, respectively. An X-ray waveguide, which was used as the collimator, was placed between the X-ray tube and an analyte, and the sizes of the entrance and exit apertures for passing X-rays of the waveguide were $0.03 \mathrm{~mm}$ in height and $1 \mathrm{~cm}$ in width. A silicon drift detector VITUS-SDD (Ketek GmbH, Munich, Germany) was used as an energy-dispersive X-ray detector. Both ends of a single fiber sample, which was placed on a quartz glass substrate with a diameter of $30 \mathrm{~mm}$ and a thickness of $5 \mathrm{~mm}$, were attached with adhesive tapes. A quartz glass substrate on which a single fiber sample was placed was tilted at $0.05^{\circ}$ to the horizontal in order to set the glancing angle of the incident X-ray beam with respect to the quartz glass substrate to $0.05^{\circ}$. A single fiber sample for the measurement was obtained from a bundle of fibers with a length of about $3 \mathrm{~cm}$ that was cut from a textile product. The angle between a single fiber sample on the quartz glass substrate and the irradiation direction of the incident X-ray beam was set to about $90^{\circ}$. Spectra of single fibers were measured in air for $600 \mathrm{~s}$ except for a spectrum of a strand of polyester fiber A, and a strand of polyester fiber A was analyzed in air for $1800 \mathrm{~s}$.

Cross sections of a strand of polyester fiber A, a single acrylic fiber, and a single nylon fiber were observed by an optical microscope ECLIPSE LV100ND (Nikon Solutions Co. Ltd., Japan) in order to estimate diameters of them, and long diameters of these single fibers were ten and several micrometers. An analyte for observing a cross section of a single fiber was prepared as follows: (1) ten single fibers obtained from a textile product were embedded in a glue; and (2) they were sliced using a microtome RM2125 RTS microtome (Leica Biosystems K. K., Germany). 


\section{Results and Discussion}

Figure 1 shows representative XRF spectra of a quartz glass substrate, strands of polyester fibers A, B, C, and D, and a white acrylic fiber. Figures $1 \mathrm{~d}$ and $1 \mathrm{e}$ show XRF spectra of three different strands of polyester fiber $\mathrm{C}$ and those of three different strands of polyester fiber D. Figure 2 shows enlarged views of Figs. 1b, 1c, 1f, and the spectrum denoted by a black solid line in Fig. 1e in the X-ray energy range from 3 to 5 keV. As shown in Fig. 1, the Si Ka line, Ar K lines, and Ta L lines were attributed to the quartz glass substrate, air containing $0.9 \%$ of $\mathrm{Ar}$, and the anode material of the X-ray tube, respectively. Spectral backgrounds in Figs. 1b-1f were as low as or slightly higher than that in Fig. 1a. This result showed that the scattering of the incident X-ray beam from a single synthetic fiber on a quartz glass substrate was weak. Although the backgrounds were low when single fiber samples were measured by using the portable TXRF spectrometer, the measured spectra were referred to as XRF spectra in this study. The Ti K lines were detected from polyester fiber A as shown in Fig. 1b. A peak was observed at around $3.65 \mathrm{keV}$ in Fig. 1b. The energy of the $\mathrm{Sb} L \alpha$ line $(3.61 \mathrm{keV})$ is near that of the $\mathrm{Ca} \mathrm{K \alpha}$ line $(3.69 \mathrm{keV})$. Therefore, when a peak is observed in the $\mathrm{X}$-ray energy range from 3.6 to $3.7 \mathrm{keV}$, determining whether this peak originates from $\mathrm{Ca}$ or $\mathrm{Sb}$ is needed. As shown in Fig. 2a, the peak observed at around $3.65 \mathrm{keV}$ in Fig. $1 \mathrm{~b}$ was lower than that of the $\mathrm{Ca} \mathrm{K} \alpha$ line in Fig. 1f, but higher than that of the $\mathrm{Sb} \mathrm{L} \alpha$ line. A tiny protrusion appeared in the $\mathrm{X}$-ray energy range from 3.8 to $3.9 \mathrm{keV}$ in the spectrum of a strand of polyester fiber A as shown in Fig. 2a, and this tiny protrusion would be attributed to the $\mathrm{Sb} \mathrm{L} \beta$ line $(3.84 \mathrm{keV})$. Therefore, we concluded that the peak observed at around $3.65 \mathrm{keV}$ in Fig. $1 \mathrm{~b}$ was a sum of the $\mathrm{Sb} \mathrm{L} \alpha$ line and the $\mathrm{Ca} \mathrm{K} \alpha$ line. The $\mathrm{Ba} \mathrm{L}$ lines were detected from polyester fiber $\mathrm{B}$ as shown in Fig. 1c, and the 
$\mathrm{S}$ and $\mathrm{Ca} \mathrm{K} \alpha$ lines also appeared in Fig. 1c. As shown in Fig. 1d, a weak $\mathrm{Ca} \mathrm{K} \alpha$ line, whose ratio of the net intensity of the $\mathrm{Ca} \mathrm{K} \alpha$ line to the value obtained by multiplying the square root of the background intensity by 10 was lower than 1 , was observed from one of three different strands of polyester fiber $\mathrm{C}$, but the Ca peak was hardly observed or not detected from the other two. As shown in Fig. 1e, a weak peak at around 4.5 $\mathrm{keV}$, whose ratio of the net intensity of this peak to the value obtained by multiplying the square root of the background intensity by 10 was lower than 1 , was detected from one of three different strands of polyester fiber $\mathrm{D}$, but this peak was not detected from the other two. The energy of the $\mathrm{Ti} \mathrm{K} \alpha$ line $(4.51 \mathrm{keV})$ is near that of the $\mathrm{Ba} \mathrm{L} \alpha$ line (4.47 keV). When the $\mathrm{Ti} \mathrm{K} \beta$ line $(4.59 \mathrm{keV})$ and $\mathrm{Ba} \mathrm{L} \beta$ line $(4.83 \mathrm{keV})$ were not observed in an XRF spectrum, determining whether a peak observed at around $4.5 \mathrm{keV}$ originates from $\mathrm{Ti}$ or $\mathrm{Ba}$ is needed. As shown in Fig. $2 \mathrm{~b}$, the energy of the weak peak observed at around $4.5 \mathrm{keV}$ in the spectrum denoted by a black solid line in Fig. 1e was higher than that of the $\mathrm{Ba} \mathrm{L} \alpha$ line in Fig. 1c, and was near that of the Ti Ka line. Therefore, we concluded that this weak peak in Fig. 1e was the $\mathrm{Ti} \mathrm{K} \alpha$ line. As mentioned above, weak $\mathrm{Ca}$ and Ti peaks were not always detected as shown in Figs. 1d and 1e. This may be attributed to inhomogeneous distributions of $\mathrm{Ca}$ and $\mathrm{Ti}$. Another possibility is that the Ca peak in Fig. 1d and the Ti peak in Fig. 1e may be due to contamination that occurred during placing a single fiber sample on the sample holder. The $\mathrm{S}, \mathrm{Cl}$, and $\mathrm{Ca} \mathrm{K} \alpha$ lines were detected from acrylic fiber as shown in Fig. 1f. Ti detected from polyester fiber A would originate from $\mathrm{TiO}_{2}$ acting as delustering agent. Antimony compounds are used as catalyst for manufacturing polyester, ${ }^{13}$ and $\mathrm{Sb}$ detected from polyester A would be due to catalyst. Biver et al. ${ }^{13}$ reported that the concentration of $\mathrm{Sb}$ in six textile products made from polyester ranged from 125 to 471 $\mu \mathrm{g} / \mathrm{g}$. Therefore, several hundred $\mu \mathrm{g} / \mathrm{g}$ of $\mathrm{Sb}$ may remain in polyester fibers when 
antimony compound is used as catalyst. $\mathrm{CaCO}_{3}$ and $\mathrm{BaSO}_{4}$ are used as filler for polymer materials. ${ }^{14}$ Therefore, $\mathrm{S}$ and $\mathrm{Ba}$ detected from polyester fiber $\mathrm{B}$ may be attributed to $\mathrm{BaSO}_{4}$, and $\mathrm{Ca}$ detected from polyester fiber $\mathrm{D}$ and acrylic fiber may originate from $\mathrm{CaCO}_{3}$. Figure 3 shows representative XRF spectra of single black synthetic fibers. As shown in Fig. 3a, $\mathrm{Ca}$ and $\mathrm{Br}$ were detected from polyester fiber $\mathrm{E}$. $\mathrm{S}, \mathrm{Ca}, \mathrm{Br}$, and $\mathrm{Ba}$ were detected from polyester fiber $\mathrm{F}$ as shown in Fig. 3b. $\mathrm{S}, \mathrm{Ca}, \mathrm{Ti}$, and $\mathrm{Cr}$ were detected from the nylon fiber as shown in Fig. 3c. Disperse dyes are used for dyeing polyester fibers. ${ }^{15}$ A disperse dye containing $\mathrm{Br}$ (Disperse Blue 291:1) was frequently detected from black polyester fibers obtained from gloves, ${ }^{16}$ and therefore the $\mathrm{Br}$ peaks in Figs. $3 \mathrm{a}$ and $3 \mathrm{~b}$ would originate from the disperse dye containing $\mathrm{Br}$. The $\mathrm{Cr} \mathrm{K}$ lines in Fig. 3c would originate from either an acid mordant dye or a chromium complex dye. These types of dyes are usually used for coloring nylon fibers. ${ }^{17}$

In this study, characteristic elements that would be attributed to materials such as catalyst, delustering agent, and dye were detected by analyzing a single synthetic fiber sample using the portable TXRF spectrometer, and the difference in the types of characteristic elements among several synthetic fiber samples was observed as shown in Figs. 1 and 3. This result shows that the difference among single fiber samples can be distinguished by only comparing the types of detected elements. However, elements whose fluorescent X-ray peaks were weak in spectra were not always detected as shown in Figs. 1d and 1e. In this study, when the net intensity of a fluorescent X-ray peak was lower than the value obtained by multiplying the square root of the background intensity by 10 , this peak was defined as a weak peak. Therefore, the difference in the types of elements whose fluorescent X-ray peaks are clearly observed should be practically employed for determining whether the origin of a single fiber sample is different from that of a sample to be compared. Elemental analysis of single synthetic 
fiber samples is performed by the portable TXRF spectrometer without influence of colors of fibers, and this portable spectrometer can be used for distinguishing the difference among single fiber samples that are not able to be distinguished by observing morphologies and colors of these samples using an optical microscope. It is difficult to distinguish the difference among single synthetic fiber samples when the types of elements whose fluorescent X-ray peaks are clearly observed are the same among these samples. In such case, these single fiber samples are needed to be analyzed by other methods such as microspectrophotometry and Raman spectroscopy for obtaining information other than elements. Sample preparation for analysis of a single fiber sample using the portable TXRF spectrometer is to place this fiber sample on a sample holder, and this simple sample preparation is suitable for performing rapid analysis of multiple fiber samples. A single fiber sample fixed on a sample holder, which has been non-destructively analyzed by the portable spectrometer, can be reused without any change for obtaining information other than elements using other analytical methods. The portable TXRF spectrometer can be applied to on-site screening for single synthetic fiber samples needed to be analyzed by other methods for performing highly accurate identification of fibers. Especially, by performing this screening method using the portable spectrometer after on-site screening for single synthetic fiber samples using an optical microscope, the number of single fiber samples that are needed to be analyzed by multiple methods will be efficiently reduced and the time required for conducting identification of fibers will be reduced.

\section{Conclusions}

In this study, XRF spectra of single synthetic fibers obtained from several textile 
products were measured by a portable TXRF spectrometer. Characteristic elements, which would originate from materials used for producing synthetic fibers, were detected. The difference among single synthetic fiber samples was able to be distinguished by only comparing the types of characteristic elements detected from each single fiber sample. A synthetic fiber sample, which is not able to be determined whether the origin of this sample is different from that of a sample to be compared by comparing the types of elements, is needed to be analyzed by other methods for identifying the difference between them. Qualitative XRF analysis using the portable spectrometer makes it possible to perform on-site screening for synthetic fiber samples needed to be analyzed by other methods in order to perform highly accurate identification of fibers.

\section{References}

1. N. Miyamoto, Y. Saito, and M. Takatsu, SEN'I GAKKAISHI, 2008, 64, 184.

2. A. Prange, U. Reus, H. Böddeker, R. Fischer, and F. P. Adolf, Anal. Sci., 1995, 11, 483.

3. R. D. Koons, J. Forensic Sci., 1996, 41, 199.

4. Y. Nishiwaki, K. Ishii, T. Takekawa, and K. Gamoh, Jpn. J. Forensic Sci. Tech., 2016, 21, 67.

5. V. Causin, Anal. Methods., 2010, 2, 792.

6. Y. Nishiwaki, S. Honda, T. Yamato, R. Kondo, A. Kaneda, and S. Hayakawa, J. Forensic Sci., 2020, 65, 1474.

7. H. Takahara, W. Matsuda, Y. Kusakabe, S. Ikeda, T. Moriyama, Y. Nishiwaki, Adv. X-Ray Chem. Anal., Jpn. , 2019, 50, 349.

8. J. V. Goodpaster and E. A. Liszewski, Anal. Bioanal. Chem., 2009, 394, 2009.

9. S. Kunimura and J. Kawai, Anal. Chem., 2007, 79, 2593.

10. S. Kunimura, S. Kudo, H. Nagai, Y. Nakajima, and H. Ohmori, Rev. Sci. Instrum., 2013, 84, 
046108.

11. M. Horiguchi and S. Kunimura, Anal. Sci., 2020, 36, 1311.

12. S. Kunimura, Y. Tokuoka, and U. Aono, Anal. Sci., 2018, 34, 1401.

13. M. Biver, A. Turner, and M. Fillela, Regul. Toxicol. Pharmacol., 2021, 119, 104824.

14. K. Nagata and K. Sanada, Journal of Network Polym., Jpn., 2015, 36, 299.

15. K. Imada, J. Text. Mach. Soc. Jpn., 2003, 56, 181.

16. T. Kato, Y. Suzuki, and M. Handa, Bunseki Kagaku, 2017, 66, 39.

17. K. Imada, J. Text. Mach. Soc. Jpn., 2002, 55, 445.

\section{Figure captions}

Fig. 1 Representative XRF spectra of (a) a quartz glass substrate, polyester fibers (b) A, (c) B, (d) C, and (e) D, and (f) a white acrylic fiber. Each XRF spectrum in Figs. 1b-1f was obtained from the measurement of a single fiber sample. In Figs. 1d and 1e, XRF spectra of three different strands of polyester fiber $\mathrm{C}$ and those of three different strands of polyester fiber D are shown. The insets in Figs. 1a, 1b, 1d, and 1e show magnified spectra in the X-ray energy range from 3 to $5 \mathrm{keV}$, and those in Figs. 1c and 1f show magnified spectra in the X-ray energy range from 2 to $4 \mathrm{keV}$.

Fig. 2 (a) Enlarged views of Figs. $1 \mathrm{~b}$ and $1 \mathrm{f}$ in the $\mathrm{X}$-ray energy range from 3 to $5 \mathrm{keV}$ and (b) those of Figs. 1c and the spectrum denoted by a black solid line in Fig. 1e in the X-ray energy range from 3 to $5 \mathrm{keV}$. A tiny protrusion, which would be attributed to the $\mathrm{Sb} \mathrm{L} \beta$ line, is indicated as a black circle $(\bullet)$.

Fig. 3 Representative XRF spectra of polyester fibers (a) E and (b) F and (c) a black nylon fiber. Each XRF spectrum in Fig. 3 was obtained from the measurement of a single fiber sample. The insets in Figs. 3a and 3c show magnified spectra in the X-ray energy range from 3 to $5 \mathrm{keV}$, and that in Fig. 3b shows magnified spectrum in the X-ray energy range from 2 to $4 \mathrm{keV}$. 

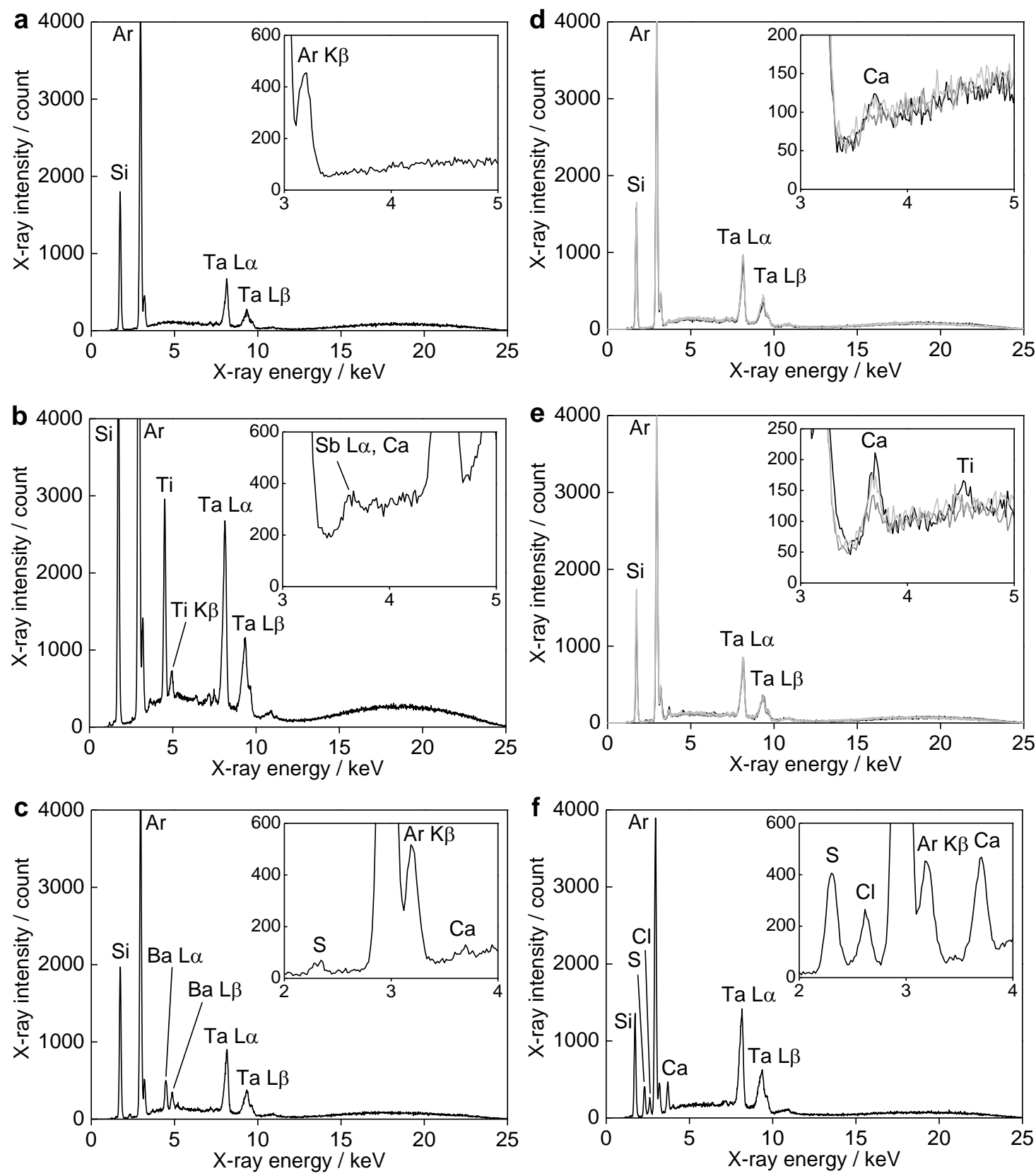
Fig. 1 Representative XRF spectra of (a) a quartz glass substrate, polyester fibers (b) A, (c) B, (d) C, and (e) D, and (f) a white acrylic fiber. Each XRF spectrum in Figs. 1b-1f was obtained from the measurement of a single fiber sample. In Figs. 1d and 1e, XRF spectra of three different strands of polyester fiber $\mathrm{C}$ and those of three different strands of polyester fiber D are shown. The insets in Figs. 1a, 1b, 1d, and 1e show magnified spectra in the X-ray energy range from 3 to $5 \mathrm{keV}$, and those in Figs. 1c and 1f show magnified spectra in the X-ray energy range from 2 to $4 \mathrm{keV}$. 

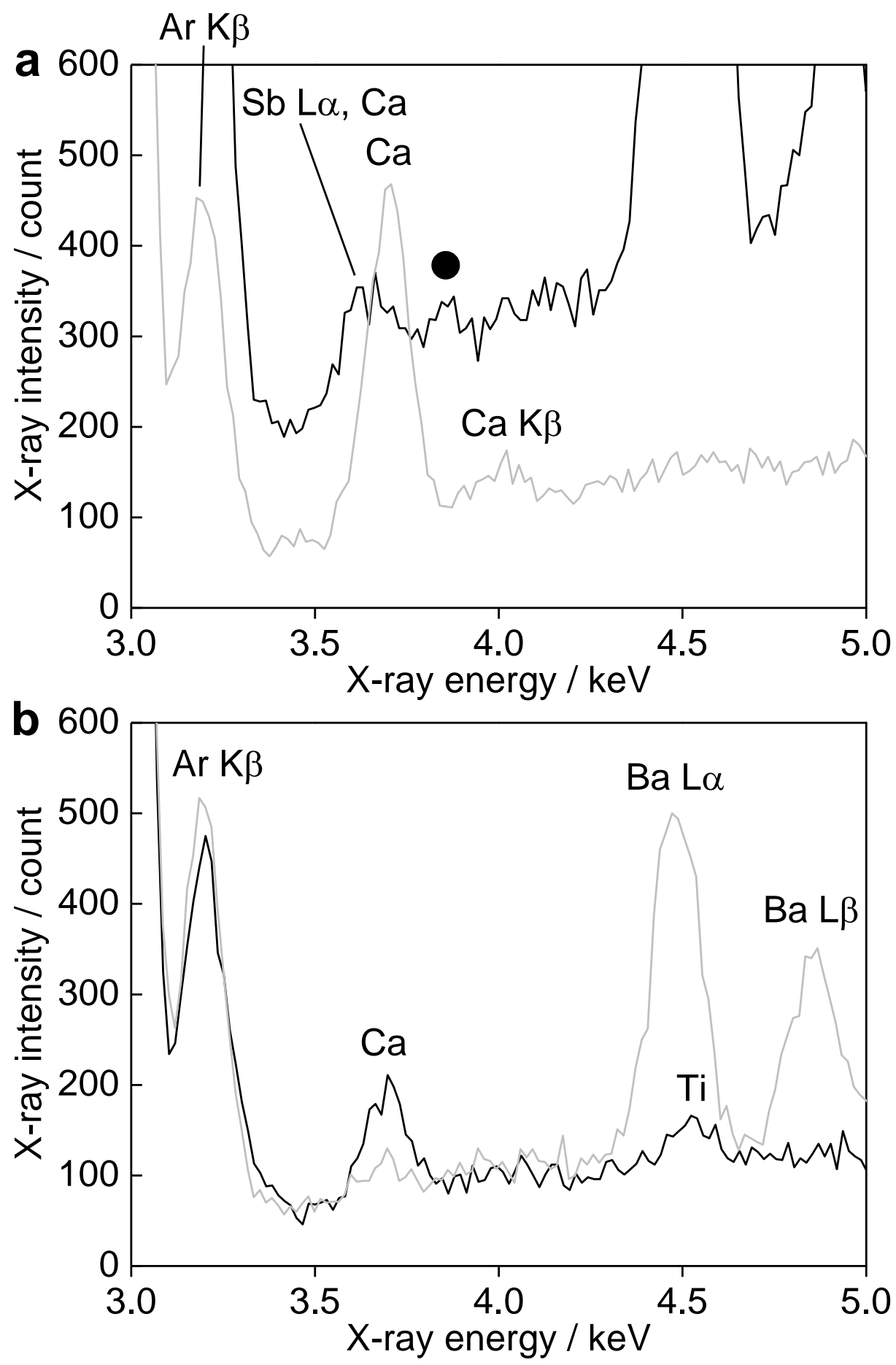

Fig. 2 (a) Enlarged views of Figs. $1 \mathrm{~b}$ and $1 \mathrm{f}$ in the $\mathrm{X}$-ray energy range from 3 to $5 \mathrm{keV}$ and (b) those of Figs. 1c and the spectrum denoted by a black solid line in Fig. 1e in the X-ray energy range from 3 to $5 \mathrm{keV}$. A tiny protrusion, which would be attributed to the $\mathrm{Sb} \mathrm{L} \beta$ line, is indicated as a black circle (•). 

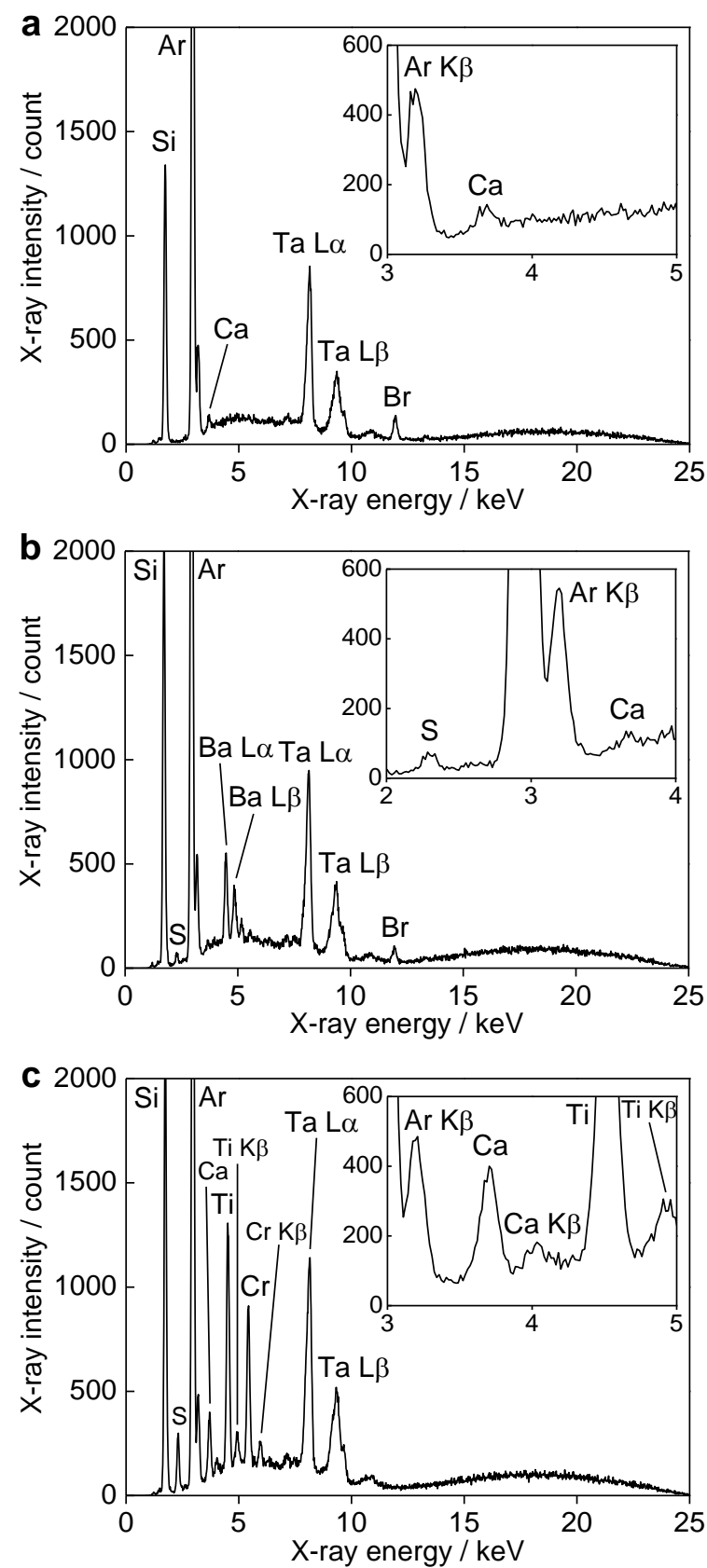

Fig. 3 Representative XRF spectra of polyester fibers (a) E and (b) F and (c) a black nylon fiber. Each XRF spectrum in Fig. 3 was obtained from the measurement of a single fiber sample. The insets in Figs. 3a and 3c show magnified spectra in the X-ray energy range from 3 to $5 \mathrm{keV}$, and that in Fig. 3b shows magnified spectrum in the X-ray energy range from 2 to $4 \mathrm{keV}$. 


\section{Graphical Index}

\section{Analysis of single synthetic fibers using a portable TXRF spectrometer}

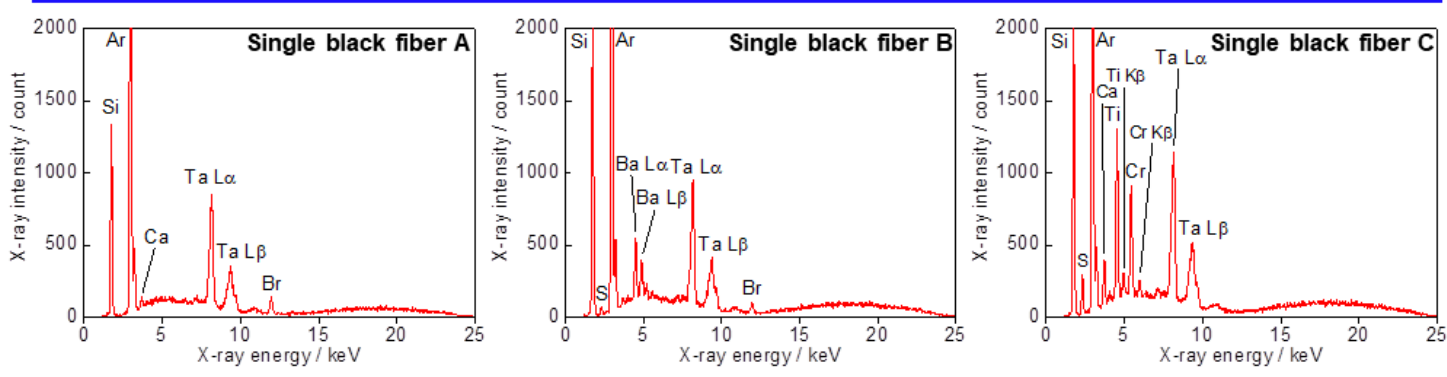

